\title{
Predicting the Intended Motion with EMG Signals for an Exoskeleton Orthosis Controller
}

\author{
Christian Fleischer, Christian Reinicke, Günter Hommel \\ Institute for Computer Engineering and Microelectronics \\ Berlin University of Technology, Germany \\ $\{$ fleischer, reinicke, hommel\}@cs.tu-berlin.de
}

\begin{abstract}
In this paper we present a method to calculate the intended motion of joints in the human body by analysing EMG signals. Those signals are emitted by the muscles attached to the adjoining bones during their activation.

With the resulting intended motion a leg orthosis will later be controled in real-time to support disabled people while walking or climbing stairs and help patients suffering from the effects of a stroke in their rehabilitation efforts.

To allow a variety of different motions, a human body model with physical properties is developed and synchronized with data recorded from the pose sensors. Computing the intended motion is performed by converting calibrated EMG signals to muscle forces which animate the model.

The algorithm was evaluated with experiments showing the calculated intended motion while climbing one step of a stair. The algorithm and the experimental results are both shown.

Index Terms-Exoskeleton, Orthosis, EMG, Rehabilitation, Intended Motion.
\end{abstract}

\section{INTRODUCTION}

EMG signals have long been studied by many researchers to analyse disabilities, anomalies or to track progress in rehabilitation. Except some early work [1], only in recent years the focus shifted to control robot arms and exoskeletons with EMG signals [2], [3]. The advantage of using EMG signals is their ability to predict the intended motion (motion the subject wants to perform but cannot in some cases) as long as the muscles are not paralyzed, but even if they are too weak to actually perform the movement. This is the main reason for choosing those signals as the interface between the subject and the orthosis aside from the intuitive handling: In our environment the orthosis (see Fig. 1) that is attached to the leg restricts the motion in the knee if the actuator is not powered. Thus the intention has to be recognized without any motion being carried out to control the actuator accordingly.

In many EMG applications (like grasping objects with a prostheses, e.g. [4]) the signals are used to toggle binary actions or recognize activation patterns [5] to control predefined trajectories. When considering full-body motions like walking or climbing stairs a more flexible input handling is desirable to allow the subject to maintain balance, step over obstacles and adapt the motion to different velocities.

Since the actuator powering the knee does not behave like a human muscle, but instead introduces a resistance to the actuated joint if it is not or only low powered, a body model has to be developed to simulate the behaviour of the joint taking into account the intended joint torque as derived from the EMG signals, influences from the adjoining bones and the external forces - as if the actuator did not exist.

The resulting motion has to be performed online by the actuator. Considering all that, two main problems have to be solved to perform intended motion prediction with EMGsignals:

- development of a body model simulating the subject,

- calibration of the EMG signals.

The body model has to cope with different points of contact with the environment and influences from parts of the subject's body that are not modeled (and thus no information about their pose is available).

Many body models have been developed (e.g. Delp et. al. [6]), driven with EMG signals and are able to calculate the resulting joint torques [7], [8], [9]. The problem when applying those models to an exoskeleton orthosis is their sometimes high complexity or the need for external sensors like force plates, dynamometers or vision systems for calibration and computation of the dynamics.

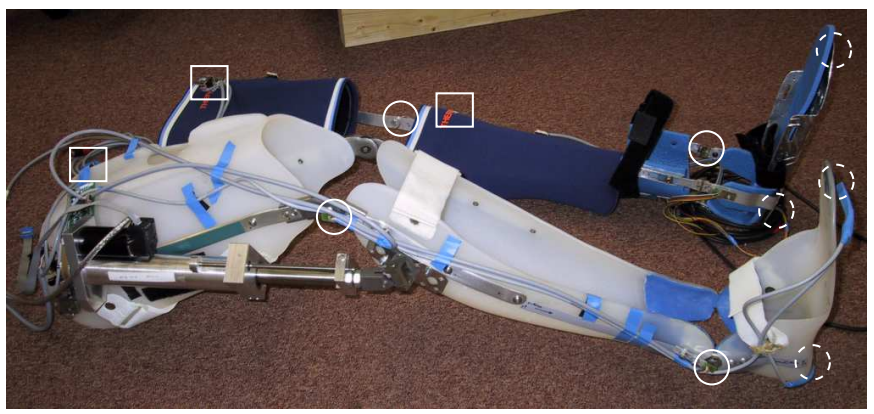

Fig. 1. This image shows the orthosis with the attached actuator and all pose sensors in the foreground (Hall sensors: solid circles, accelerometers: squares, floor contact sensors: dashed circles). In the background the lightweight orthosis for the left leg can be seen.

Our approach is to derive a more simplified body model, taking into account only the most important properties of the subject. All varying parameters should be calculated through the sensors attached to the orthosis to assure consistency of the calibration with the sensor readings during interaction with the orthosis.

In our case it is not necessary that calculated muscle forces match actual forces in the subject's body exactly as in 
clinical diagnosis. For our purpose it is essential that the body model performs the same motions when the EMG signals are applied, because they are interpreted as the desired motion and will be executed.

The calibration of the EMGsignals is very important since they are different for every subject and from day-to-day, depending on numerous factors like moisture on the skin, muscle fatigue and blood circulation [10]. The problem has been approached for applications like this by ourselves as presented in [11], but an improved version is presented here allowing co-contraction of muscles and taking into account ground reaction forces.

As can be seen in Fig. 1, the orthosis covers the right thigh, shank and foot. Currently only intention reading and force support for the knee joint has been realized.

\section{FRAMEWORK}

This section describes the framework in which the motion prediction algorithm is running. Please refer to Fig. 2 for an overview of the whole system.

\section{A. Measurement System}

The measurement system used for the algorithm consists of two groups of sensors: The EMG sensors to read the muscle activity and the pose sensors to get the current state of the subject.

The EMG sensors are placed on top of selected muscles responsible for flexing and extending the knee: the $\mathrm{M}$. semimembranosus and M. vastus medialis [12], [13]. Many other muscles cooperate during those motions but we have chosen those as they are most clear and simple to record and largely contribute to the resulting torque in the knee [14]. The signals are sampled with $1 \mathrm{kHz}$ from DelSys 2.3 Differential Signal Conditioning Electrodes [15] with an built-in gain of $1000 \frac{V}{V}$ and a bandpass filter from $20-450 \mathrm{~Hz}$. In software the offsets of the signals are eliminated, the resulting signal rectified and lowpass filtered with $4 \mathrm{~Hz}$ to approximate activation envelopes of the muscles.

Ankle and knee angles are measured in sagittal plane on both legs with Philips KMZ41 Hall sensors [16] and the thigh and trunk angles with accelerometers ADXL210 from AnalogDevices Inc. [17] (as described in [18], [11]). All sensors measuring the current pose are attached to the orthosis as shown in Fig. 1 except the torso sensor on the belly-plate which is not shown. In addition to that, pressure sensors are attached under the heel and footpad on both feet to detect floor contact (binary result: contact / no contact). All sensors are sampled with $1 k H z$.

\section{B. Signal Flow}

As mentioned in the introduction, the intended motion of the subject should be analysed to let the human control the orthosis (Fig. 2). First, the current pose of the subject is read from the pose sensors attached to the limbs and fed into the biomechanical model. To determine the intended motion of the joints within the orthosis, the EMG signals from the appropriate muscles are recorded, converted into muscle forces and also fed into the biomechanical model. The model now calculates the knee torque from the muscle forces and computes the resulting acceleration for the knee joint by regarding all joint torques and the ground reaction forces. The knee acceleration is interpreted as the desired motion and passed to the motion controller. Depending on the actual implementation of the controller, the acceleration can be integrated once or twice.

To be able to use the EMG-to-force function, parameters of the function have to be calibrated. This is performed in the block calibration: The biomechanical model calculates the knee torque (torque that must have been active in the knee to produce the current motion) using inverse dynamics. This torque, together with the corresponding EMG values of all recorded muscles, are fed into the block calibration to optimize the parameters of the EMG-to-force functions. The computed parameters are then passed to the EMG-to-force functions again.

\section{Computing the Intended Motion}

Computing the intended motion is based on interpreting the results of the online simulation of the subject's body.

\section{A. Body Model}

The human body model of the simulation consists of two legs with feet, shanks, thighs and the torso. All limbs and the torso are modelled as rigid bodies (rectangular parallelepipeds) connected with swivel joints that can rotate in sagittal plane only. Body masses for the torso, thighs, shanks and feet are calculated as fixed fractions of the total body weight $\left(m_{\text {total }}=88 \mathrm{~kg}\right)$ of the subject (the figures can be found e.g. in [19]). Body dimensions are taken from our subject. Two muscles $M_{f}$ (flexor) and $M_{e}$ (extensor) have been added to produce the corresponding forces $F_{M_{f}}$ and $F_{M_{e}}$ to allow flexion and extension of the knee. The points of origin $\vec{O}_{f}, \vec{O}_{e}$ and insertion $\vec{I}_{f}, \vec{I}_{e}$ of the muscles are fixed and have been chosen by hand in analogy to human anatomy.

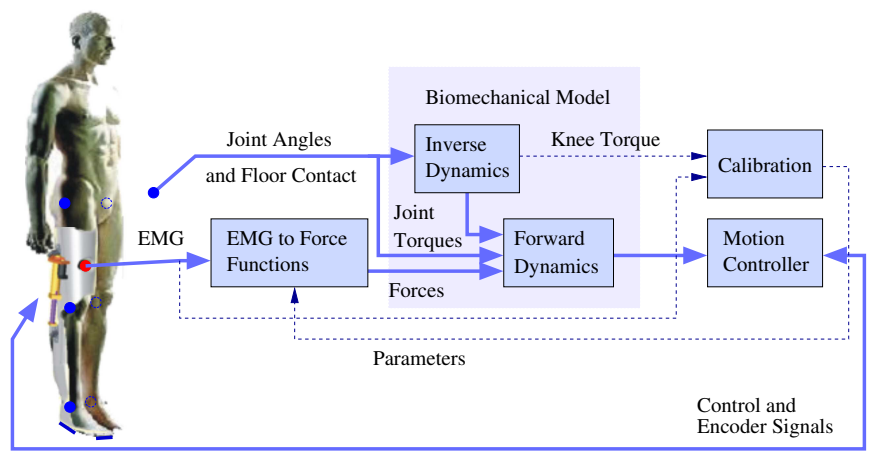

Fig. 2. The figure shows the data flow within the system. The solid lines show the flow in normal operation mode, the dotted lines mark additional signals during EMG calibration. 
The dynamic equations of the model were derived using Kane's formalism [20]

$$
\mathbf{M}(\mathbf{q}) \dot{\mathbf{u}}=\mathbf{f}(\mathbf{q}, \mathbf{u})+\mathbf{T} \times \mathbf{g}(\mathbf{q})
$$

where

- q: vector of generalized coordinates, which are joint angles $q_{h i p}^{r}, q_{k n e e}^{r}, q_{\text {ankle }}^{r}, q_{\text {hip }}^{l}, q_{k n e e}^{l}, q_{\text {ankle }}^{l}, q_{\text {torso }}$ and coordinates of the reference point $q_{\text {pelvis }}^{x}, q_{\text {pelvis }}^{y}$ with the $x$-axis parallel and the $y$-axis perpendicular to the ground in the sagittal plane),

- $\mathbf{u}$ : vector of generalized velocities, with $\dot{\mathbf{q}}=\mathbf{u}$ (time derivative in the Newtonian reference frame),

- M (matrix function): specifies mass distribution,

- f (vector function): adds inertial forces and gravity,

- T: takes into account all torques in the joints as a result of the muscle forces applied: $t_{h i p}^{r}, t_{k n e e}^{r}, t_{a n k l e}^{r}$, $t_{\text {hip }}^{l}, t_{\text {knee }}^{l}, t_{\text {ankle }}^{l}$ together with the external forces $F_{x}^{l}$, $F_{y}^{l}, F_{x}^{r}, F_{y}^{r}$ applied to the left and right ankles in $x-$ and $y$-direction, $F_{x}^{h}$ applied to the hip and the torques applied between the reference frame and the feet $T^{l}, T^{r}$,

- $\mathbf{g}(\mathbf{q})$ : nonlinear function representing the current system configuration and geometry.

The dynamic equations (1) were generated with the symbolic manipulation tool AUTOLEV [21] resulting in a system of nine equations. The script for the system description and equations generation can be received on request.

The body model is used in two different ways: the forward dynamics and the inverse dynamics (Fig. 2). The inverse dynamics block solves the Eqs. 1 for elements of $\mathbf{T}$ : the torques in the joints (as contributed by the muscles, not by external forces or other body parts) and some external forces depending on the current contact configuration of the feet with the ground:

- only left foot: $F_{x}^{l}, F_{y}^{l}$ and $T^{l}$,

- only right foot: $F_{x}^{r}, F_{y}^{r}$ and $T^{r}$,

- both feet: $F_{y}^{l}, F_{y}^{r}$ and $F_{x}^{h}$

(is symmetric for both legs and avoids singularities when feet are close together).

The other elements of $\mathbf{T}$ are set to 0 . The accelerations $\dot{\mathbf{u}}$ needed to perform the inverse dynamic computation are numerically derived from the values of the pose sensors with $\Delta t=30 \mathrm{~ms}$. After this computation the contributions of the mentioned torques and forces that result in the current motion are known.

The forward dynamics block takes the current system state $\mathbf{S}(\mathbf{t})=(\mathbf{q}(\mathbf{t}), \mathbf{u}(\mathbf{t}))^{T}$ and applies all internal and external torques (except for the actuated knee $t_{k n e e}^{r}$ ) and external forces as calculated by the inverse dynamics, assuming they are all constant for a short period of time. After that, Eq. 1 is solved for $\dot{\mathbf{u}}$ as the solution of the forward dynamics.

The torque of the actuated knee joint $t_{k n e e}^{r}$ is calculated by converting the EMG values to activations as proposed in [22][7] and scaling them to the maximum force (EMG-toforce function):

$$
F_{M_{j}}(t)=\frac{e^{A_{j} u_{j}(t)}-1}{e^{A_{j}}-1} \cdot f_{j, \max }
$$

where $F_{M_{j}}(t)$ is the force produced by the muscle $j$ with $j=1 \ldots N$ ( $N$ : number of recorded muscles), $u_{j}(t)$ the postprocessed and scaled EMG signal at time $t$ of muscle $j$ and $A_{j}$ the non-linear shape factor. $A_{j}$ was limited to $-10<A_{j}<0$ in our setup. The scale for $u_{j}(t)$ is set to $s_{j}=1 / u_{j, \max }$ where $u_{j, \max }$ is the maximum recorded post-processed EMG signal during calibration with the corresponding maximum force $f_{j, \max }$.

The total knee joint torque $t_{k n e e}^{r}$ is calculated as a sum of all force contributions of the muscles spanning the knee joint with

$$
t_{k n e e}^{r}(t)=\sum_{j=1}^{N}\left(\left(\vec{I}_{j}^{R}-\vec{J}^{R}\right) \times \frac{\vec{I}_{j}^{R}-\vec{O}_{j}^{R}}{\left|\vec{I}_{j}^{R}-\vec{O}_{j}^{R}\right|} \cdot F_{M_{j}}\right),
$$

where $\vec{J}^{R}$ is the vector to the knee joint and $\vec{O}_{j}^{R}$ and $\vec{I}_{j}^{R}$ the points of origin and insertion in the reference frame.

Co-contraction is allowed during normal operation mode but is not handled explicitly (e.g. by changing properties of the knee joint).

\section{B. EMG-to-Force Calibration}

Since EMG signals vary very much even for the same subject from day-to-day, the parameters $f_{j, \max }$ and $A_{j}$ from the EMG-to-force functions in Eq. 2 have to be calibrated.

The optimization procedure is split into three parts that are executed online while the motions are performed by the subject:

1) gathering of data into special tables,

2) checking if optimization for a muscle is feasible,

3) optimizing parameters of one muscle at a time.

The basic idea was published by us in [11], but an improved version is presented here, taking into account co-contraction of muscles, ground reaction forces and reducing computation time by using inverse dynamics: For every muscle $m=$ $1 \ldots N$ one table exists. Every entry of those tables contains EMG signals of all muscles at time $t_{i}$ and the resulting knee torque $t_{k n e e}^{r}\left(t_{i}\right)$ as computed by the inverse dynamics. The entries of table $m$ are indexed by the activation of muscle $m$ for reading and writing.

As a result, all tables will contain unique information about different levels of muscle activations and resulting knee torques. Since the entries of the tables are indexed by activations of different muscles and not by time, redundancy of information is kept low, minimizing the costs of the optimization independent of the complexity of the necessary motion.

Before the optimization of a specific muscle can be performed, three conditions have to be met, otherwise the calibration is postponed:

1) the data table contains more entries than $c_{\min }=0.6$. $T_{\max }$ (avoids calibration without sufficient data), 
2) $c_{\text {new }}=0.8 \cdot T_{\max }$ of the non-empty entries must have been replaced since the last calibration (avoids repeated calibration without updated information),

3) all antagonistic muscles that have an activation of more than $c_{u}=0.25 \cdot u_{j, \max }$ of muscle $j$ that should be calibrated have to be already calibrated (omits unwanted bad optimizations due to missing counteraction in case of co-contraction).

$T_{\max }$ is the maximum number of entries in the table expected to be filled during the considered motion. But it is possible to resize the table at run-time if needed.

The optimization itself is performed by minimizing the error-function

$$
E=\sum_{i=1}^{T}\left(t_{k n e e}^{r, i n v}(i)-\sum_{m=1}^{N} t_{k n e e, m}^{r, e m g}(i)\right)^{2},
$$

where $t_{k n e e}^{r, i n v}$ is the torque as computed by the inverse dynamics and stored in the table and $t_{k n e e, m}^{r, e m g}$ is the torque contribution of muscle $m$ to the total knee torque. $i$ denotes the $i$-th non-empty table entry, $T$ the total number of nonempty entries.

During the optimization only the parameters of the currently considered muscle are optimized, all others are evaluated together with the corresponding EMG signals to account for co-contraction. This greatly reduces the dimension of the optimization but makes a repeated optimization necessary as soon as parameters from other muscles are changed.

In contrast to the previous version the calibration presented here is reduced to optimizing an exponential function by utilizing inverse dynamics.

Since the paramaters can be bound to

$$
\begin{gathered}
0<f_{j, \max }<f_{\max } \\
A_{\min }<A_{j}<0
\end{gathered}
$$

with $f_{\max }=10000 \mathrm{~N}$ and $A_{\min }=-10$ as experimentally determined, fast and stable optimization by subspace search can be performed.

Thinking of the two parameters of a muscle as one dimension, the repeated optimization can be seen as the implementation of a direction set algorithm [23]: The optimum is searched along one dimension. If found, the algorithm continues with the following dimension cycling through all of them until a global criterion is met that identifies a local optimum.

Fig. 3 shows two calibration curves from the experiments described in Sec. IV.

\section{Motion Controller}

The motion prediction is based on evaluating the body model with the calibrated EMG signals.

In every iteration of the algorithm the current state of the body model is synchronized with the state of the subject as read from the pose sensors. To all joints except the actuated right knee joint the torques are applied as calculated by the inverse dynamics, assuming that the muscle forces are constant for the short period of one iteration. Additionally, the similar computed ground reaction forces are applied to the model. The EMG-to-Force functions are evaluated with the current EMG signals resulting in the desired force contributions of the muscles to the total knee torque.

Computing the forward dynamics of the body model results in solutions for the angular accelerations $\dot{\mathbf{u}}$ for all joints and an acceleration of the reference point.

Since only the knee is actuated and all joints of the model are synchronized in every step, calculating $\dot{u}_{k n e e}^{r}$ is sufficient here. Depending on the actual implementation of the lowlevel motion controller $\dot{u}_{k n e e}^{r}, u_{k n e e}^{r}$ or $q_{k n e e}^{r}$ can be used as an input.

\section{EXPERIMENTS}

To evaluate the behaviour of the algorithm more easily, all experiments have been performed with healthy patients. The example experiment presented here was performed in an upright standing position. The actuator was not attached.

During the initial calibration phase the subject was standing on the left leg and the right knee was bent backwards to 63 degrees to record EMG signals of the knee flexor. After that, one step of a stair was climbed, right leg first, to record the signals of the knee extensor.

This simple experiment tests the most important aspects of the algorithm: motion of all limbs, various points of contact during single- and double-support phase, swing-phase of the right leg, co-contraction of knee flexor and extensor muscles and co-activation with other muscles that are not recorded.

The calibration of the muscle parameters was divided into three steps. The first step was performed after the backward motion with the knee flexor. Since the maximum activation of the knee extensor was below the flexor activation threshold $c_{u, f l e x}$ during this motion, the calibration procedure started after having gathered enough data. Fig. 4 shows the results of the optimization of the knee flexor parameters. Applying the
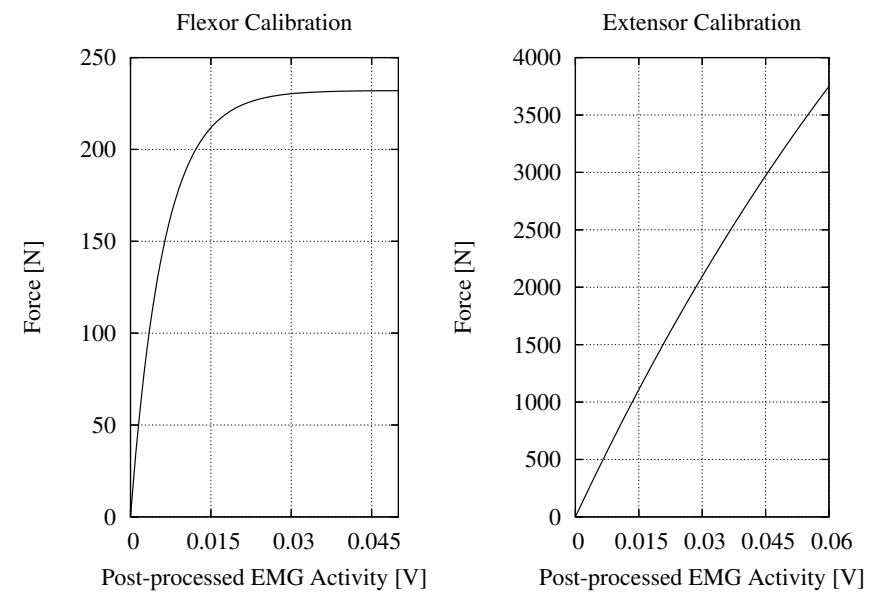

Fig. 3. This diagram shows the final results from the calibration with $A_{\text {flex }}=-8.13, f_{\text {flex } \max }=232 \mathrm{~N}\left(\right.$ at $\left.u_{\text {flex }, \max }=0.05 \mathrm{~V}\right)$ and $A_{\text {ext }}=-0.47, f_{\text {ext, } \max }=3750 \mathrm{~N}$ (at $\left.u_{\text {ext }, \max }=0.06 \mathrm{~V}\right)$. 
EMG signals to the body model results in a torque-curve very similar in shape and magnitude to the torque-curve calculated by the inverse dynamics (see Sec. V).

Step two was performed after stepping up the stair. As can be seen in the lower part of Fig. 5 both muscle groups are active during this motion resulting in the calibration of the knee extensor parameters while applying the parameters from the previous calibration to the knee flexor.

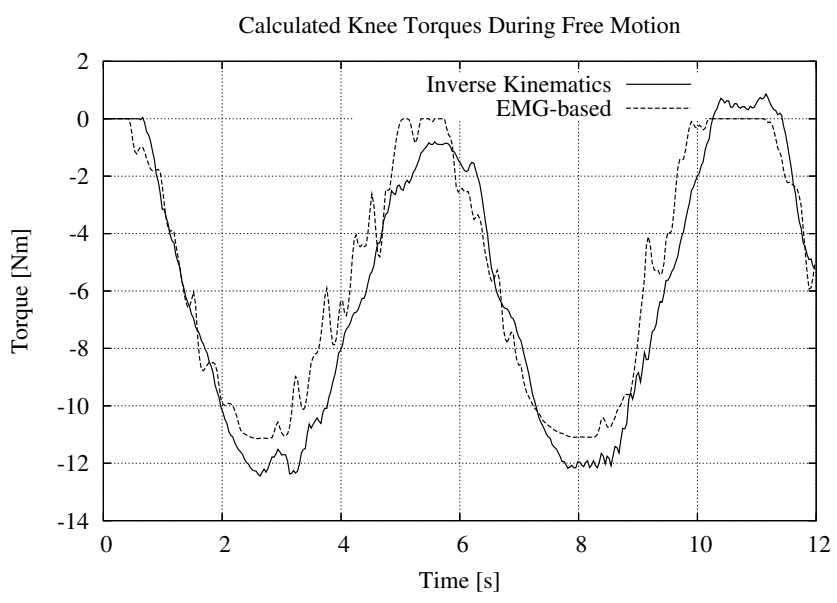

Fig. 4. This diagram shows the knee torque as calculated through inverse dynamics compared to the torque resulting from the EMG signal evaluation (after having calibrated the EMG-to-Force parameters of the knee flexor).

In the final step parameters of both muscles have been repeatedly optimized without the need of acquiring new data. This was necessary since results modify each other due to cocontraction. This procedure was terminated when the change in parameters was sufficiently small.

Fig. 3 shows the final calibration curves for both muscles and the upper part of Fig. 5 shows the torque-curves during stair-climbing after the calibration was completed. It begins when the right foot is lifted from the ground. The section between the dashed lines indicate the double support phase before the left foot is lifted and also put on the same step.

As can be seen there is an abrupt change and a high peak in the knee torque as calculated by the inverse dynamics at $t=4.62 \mathrm{~s}$. At this point a change in contact configuration occurs: The left foot is raised from the ground.

In theory, if the motion is completely tracked with head, arms and upper torso (or the torso has to be stiff) and if the pose sensors are accurate enough, an abrupt change should not occur. Unfortunately this is not the case with our experimental setup. Motion artifacts due to inertial accelerations and errors in calibration of the accelerometers attached to the torso and thighs result in inaccurate angle readings. Especially for the heavy torso this leads to wrong torque calculations in the joints for some configurations.

Furthermore, during double support phase with the feet apart no calibration data can be gathered (region between the dashed lines in Fig. 5). In this configuration the inverse dynamics cannot be calculated. The system is redundant and no unique solution exists: Changes in the muscle forces of the knee can be compensated through changes of muscle forces in the right hip and ankle joints and the left leg without performing any motion. This is also true for the forward dynamics, but since only information about EMG activity is gathered from the knee muscles and all other muscle forces are assumed to be constant, an approximated desired motion can be calculated. This desired torque can be seen in Fig. 5. In this experiment, the double support phase lasts from $3.56 s \leq t \leq 4.58 s$.

Before and after the double support phase, a good correlation of the two torque-curves can be seen expressing the performance of the body model and the consistent calibration for both muscles.

\section{RESUlTS AND Discussion}

As can be seen in the Fig. 4 and 5 the torque-curves show the prediction of the intended motion. The shape of the curves correlate very well, although sometimes the amplitudes of the curves differ. This is not a major problem for the desired application: Since the subject wearing the orthosis is inside the control loop of the system, he or she can increase or decrease the muscle activity a little to adapt to the circumstances. This is what healthy persons do every day and is part of the necessary rehabilitation for injured persons.
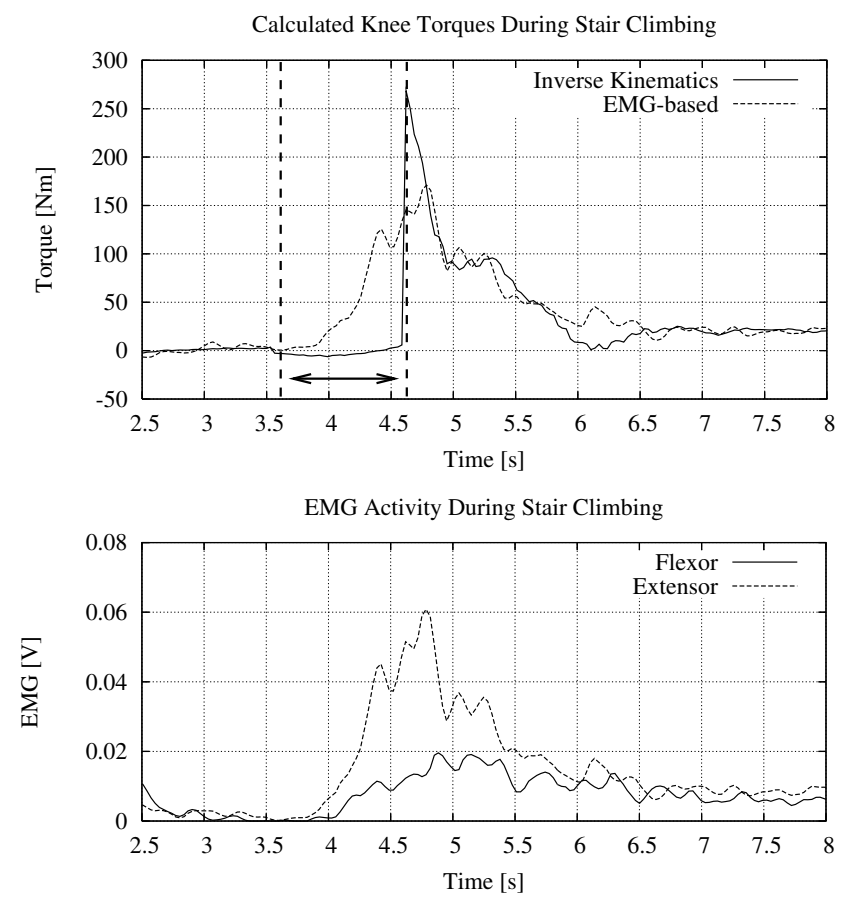

Fig. 5. In this diagram the knee torques resulting from the calibrated EMG signals for the flexor and extensor together with the torque from the inverse dynamics are shown. The region between the dashed lines indicates the double support phase. In the lower part of the diagram, the EMG activity of both muscles can be seen. 
It is more important that through the filter and data-processing chain no significant displacements in time occur. For practical experiments with the actuator attached, a time-displacement would introduce a delay while executing the desired motion. That would let the orthosis react slowly to the muscle activity.

The results also show the possibility to use one muscle out of a group to represent the activity of the whole group for a specific motion. Unfortunately, preliminary tests with different kind of motions have shown that it will be necessary to incorporate more knee flexors and extensors to handle a range of different motions.

Looking at the sensor system it has to be mentioned that a good calibration and accurate readings are very important. Otherwise inconsistencies between EMG activities and recorded motions can occur which cannot be handled easily by the calibration algorithm.

A prerequisit for all experiments is the ability of the subject to control the muscle activations to balance during movements. The natural muscle force does not have to be sufficient. But due to the limited actuated degrees of freedom of the orthosis, algorithms to maintain postural stability can be hardly applied at the moment.

\section{FUTURE WORK}

In this paper an approach has been presented that allows to calculate the intended motion of a subject wearing a lower extremities orthosis by evaluating EMG signals from certain muscles.

The next steps of research will be to experiment with the actuator attached and powered to get a feeling for the force feedback effects. It has to be examined if adding more properties to the muscles improve the prediction or if further simplifications can be made.

To allow a larger variety of motions, more muscles from the knee extensor and flexor groups have to be interpreted as our preliminary experiments have shown. This most certainly introduces a new problem of crosstalk between neighbouring muscles that has to be eliminated with algorithms like blind source separation to discriminate the different activities.

It has to be evaluated if real force support with the orthosis is possible by multiplying the output of the EMG-to-ForceFunction with a value $s>1.0$. In theory the EMG activity for one and the same motion should be reduced if no other factors interfere.

The handling of erroneous or incomplete data from the pose- and contact-sensors has to be improved to allow reliable computation in difficulty situations, e.g. when the subject is leaning to the wall or is supporting herself or himself with a handrail.

Other steps include first experiments with patients to optimize the algorithms for weak EMG signals and test the performance of the calibration. One open point here is that some patients might not be able to perform the motions needed for the calibration.
It has to be examined if this can be done with the aid of a therapist or if alternative methods of generating the parameters have to be developed. Despite of all those topics that need to be addressed, hopefully in the near future this system will provide an intuitive and easy-to-use interface for an exoskeleton orthosis.

\section{REFERENCES}

[1] S. Lee and G. N. Sridis, "The control of a prosthetic arm by EMG pattern recognition," IEEE Transactions on Automatic Control, vol. AC29, no. 4, pp. 290-302, 1984.

[2] O. Fukuda, T. Tsuji, H. Shigeyoshi, and M. Kaneko, "An EMG controlled human supporting robot using neural network," in Proceedings of the IEEE/RSJ Int. Conf. on Intelligent Robots and Systems, 1999, pp. 1586-1591.

[3] S. Morita, T. Kondo, and K. Ito, "Estimation of forearm movement from EMG signal and application to prosthetic hand control," in Proceedings of the IEEE Int. Conf. on Robotics \& Automation, 2001, pp. 3692-3697.

[4] Y. M. Matthew DiCicco, Lenny Lucas, "Comparison of control strategies for an emg controlled orthotic exoskeleton for the hand," in Proceedings of the 2004 IEEE International Conference on Robotics and Automation, 2004, pp. 1622-1627.

[5] A. K. Koji Ito, Toshio Tsuji and M. Ito, "Emg pattern classification for a prosthetic forearm with three degrees of freedom," in IEEE International Workshop on Robot and Human Communication, 1992, pp. 69-74.

[6] S. Delp, J. Loan, M. Hoy, F. Zajac, E. Topp, and J. Rosen, “An interactive graphics-based model of the lower extremity to study orthopaedic surgical procedures," IEEE Transactions on Biomedical Engineering, vol. 37, no. 8, pp. 757-767, Aug. 1990.

[7] T. F. B. David G. Lloyd, "An emg-driven musculoskeletal model to estimate muscle forces and knee joint moments in vivo," vol. 36, pp. 765-776, 2003.

[8] T. S. B. Kurt Manal, "A one-parameter neural activation to muscle activation model: estimating isometric joint moments from electromyograms," Journal of Biomechanics, vol. 36, pp. 1197-1202, 2003.

[9] L. M. David Amarantini, "A method to combine numerical optimization and emg data for the estimation of joint moments under dynamic conditions," Journal of Biomechanics, vol. 37, pp. 1393-1404, 2004.

[10] J. V. Basmajian and C. J. De Luca, Muscles Alive: Their Functions Revealed by Electromyography. Williams \& Wilkins, 1985.

[11] C. Fleischer, K. Kondak, C. Reinicke, and G. Hommel, "Online calibration of the emg force relationship," in Proceedings of the IEEE/RSJ Int. Conf. on Intelligent Robots and Systems, 2004, pp. 1305-1310.

[12] E. F. Delagi and A. Perotto, Elektromyographie der Extremitäten. Thieme, Stuttgart, 1989.

[13] D. Preston and B. Shapiro, Electromyography and Neuromuscular Disorders. Butterworth-Heinemann, 1997.

[14] W. Platzer, Taschenatlas der Anatomie. Thieme, 2003.

[15] DelSys, Inc., http://www.delsys.com.

[16] Royal Philips Electronics, http://www.philips.com/.

[17] Analog Devices, Inc., http://www.analog-devices.com.

[18] A. T. M. Willemsen, C. Frigo, and H. B. K. Boom, "Lower extremity angle measurement with accelerometers - error and sensitivity analysis," vol. 38, no. 12, 1991.

[19] D. A. Winter, Biomechanics and Motor Control of Human Movement. John Wiley \& Sons, Inc., 1990.

[20] T. Kane and D. Levinson, Dynamics OnLine: Theory and Implementation with Autolev. Kane Dynamics, Inc., 2000.

[21] Kane Dynamics,Inc., http://www.autolev.com.

[22] S. M. J.R. Potvin, R.W. Norman, "Mechanically corrected emg for the continuous estimation of erector spinae muscle loading during repetitive lifting," European Journal of Applied Physiology and Occupational Physiology, vol. 74, pp. 119-132, 1996.

[23] W. T. V. William H. Press, Saul A. Teukolsky and B. P. Flannery, Numerical Recipies in C++. Cambridge University Press, 2002. 